\title{
Analysis on loudness of exhaust noise and improvement of exhaust system based on structure-loudness model
}

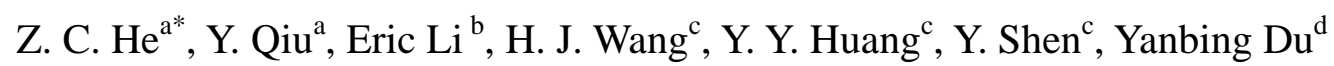 \\ ${ }^{a}$ State Key Laboratory of Advanced Design and Manufacturing for Vehicle Body, Hunan University, \\ Changsha, 410082 P. R. China \\ ${ }^{b}$ School of Science, Engineering \& Design, Teesside University, Middlesbrough, UK \\ ${ }^{c}$ SAIC-GM-Wuling Automoblie CO. LTD, Liuzhou, 545007 P. R. China \\ ${ }^{d}$ Tangsteel Technology Center of Hebei Iron and Steel, Tangshan, Hebei, China
}

\begin{abstract}
The structure of exhaust system influences the loudness of exhaust noise significantly. This paper indicates the impact of the structure parameters of exhaust system on the loudness of exhaust tail noise and presents an evaluation model to conduct the improvement of exhaust system for improved loudness. Firstly, the twenty-seven structure samples are designed by orthogonal experiment and exhaust tail noises of those are obtained by simulation. The accuracy of simulation is also validated by the experiment successfully. Then the structure-loudness model is developed by the radial basis function (RBF) network technique. Subsequently, the contributions and main effects of the structure parameters on loudness are indicated in this work. Finally, based on the structure-loudness model, the robust optimum design for improved loudness is studied by the adaptive simulated annealing (ASA) algorithm. The result of optimization certifies that the structure-loudness model is useful for the optimization. The method applied in this paper can be feasible for other psychoacoustic metrics.
\end{abstract}

Key words: sound quality; exhaust tail noise; structure-loudness model; robust optimization

\footnotetext{
* Corresponding author. Tel./fax: +86 73188822051.

E-mail address: hezhicheng815@hnu.edu.cn (ZC He);
} 


\section{Introduction}

The modern vehicle is not only a means of transportation but also a part of human's living space. Therefore, customers are paying more and more attention to the comfort characteristic of vehicle, especially the NVH (Noise, Vibration and Harshness) performance. Recently, the sound quality of interior noise of car is identified as a major component in the overall quality of vehicle by the passengers and thus the sound quality is more concerned. Over the last few decades, a significant amount of studies has been conducted to develop and apply new approaches for the evaluation of sound quality [1-3]. Lately, Volandri et al. investigated the subjective and objective evaluations of the sound quality of power window noise and analyzed the correlation between them [4]. A model of psychoacoustic sportiness was developed by Kwon et al. to describe the "sporty" of interior noise [5]. Although the evaluation of sound quality can reflect the perception of interior noise of car, it offers little guidance on the improvement of acoustic characteristics. For the sake of optimization of the sound quality of interior noise, the main noise sources are the necessary evils that need to be dealt with. For example, the sound quality of interior noise was controlled through the usage of biodegradable and recyclable natural materials which are low-cost and light-weight by Singh and Mohanty [6]. The exhaust tail noise possesses a principal component of the interior noise and the corresponding psychoacoustic metrics have an important impact on vehicle sound quality perception. Among all the psychoacoustic metrics of exhaust tail noise, the loudness is the main constituent of the sound quality.

The loudness is calculated by the sound pressure level of exhaust tail noise 
which is determined by the exhaust system. In exhaust system, the gas produced by engine recurrently passes through exhaust system to the atmosphere, generating the exhaust tail noise [7]. Compared with other noises composing the vehicle interior noise, the most apparent characteristic of exhaust noise is the impulsive influenced by the engine. Lighthill pointed out that the flow noise through a valve contains acoustic monopoles, dipoles and quadrupoles [8]. Zhao and Shi et al. indicated that the impulsive exhaust gas flows through the valves of engine, generating the acoustic source of exhaust noise which is briefly the acoustic monopoles and quadrupoles [9-11]. After passing the exhaust system, the gas is finally concentrated at the exhaust tail pipe orifice. An important component of exhaust system is the silencer which is installed to eliminate the exhaust tail noise. The silencer can partly prevent the generation of noise and impair the noise generation in the previous path [12-14]. It can be inferred that the acoustic characteristic of exhaust tail noise is determined by the exhaust system. That is to say, the outstanding quality of exhaust system can reduce the sound pressure level of tail noise magnificently, leading to a more visible improvement of the loudness of noise.

In the past, a lot of attentions have been paid to the structure optimization of exhaust system for better acoustic performance. Ranjbar investigated passive noise reduction methods in muffler and conduct an experimental examination [15]. Zhang et al. optimized the exhaust system of the diesel through computer analysis engineering and made the loss of exhaust noise more than $10 \mathrm{~dB}$ [16]. Steblaj et al. proposed an adaptive silencer and improved the transmission loss obviously [17]. However, little 
literature deals with the optimum design for improved loudness owing to the indeterminate relationship between the structure of exhaust system and loudness of noise. In order to optimize the structure, the correlation between them should be understood.

Regarding the optimization of exhaust system, it also should be noted that in the practical project, there are some inevitable errors caused in the structure manufacture resulting in huge deviation of system response. It is necessary to take the structure uncertainty into account in the process of structure design and sound quality optimization. A large number of studies about robust design have been conducted. For example, Apley et al. developed a methodology based on a Bayesian framework to quantify the effect of interpolation uncertainty on the robust design [18]. Zhang et al. proposed a RO framework to avoid infeasible robust optimal design with the consideration of parameter uncertainty [19]. Son presented a novel approach to ascertain the stability and robustness of the nonlinear dynamic system [20].

In traditional work, the correlation between the parameters of structure and sound pressure level of noise has attracted a lot of attention. However, different from previous studies, the relationship between the loudness of exhaust tail noise which is an evaluation metric of sound quality and the structure parameters of exhaust system is focused in this paper. The outline of this work is as follows. Firstly, based on a sample exhaust system, different samples which contain the parameters of structure were designed by the orthogonal method. Subsequently, the exhaust tail noises of samples were simulated and the results of the loudness of these noises were calculated. 
With the experiment verification, it was proved that the simulations have a very good accuracy and can be used to succedent investigation and improvement. According to the samples, the contributions and main effects of structure parameters were analyzed. Moreover, the structure-loudness model was developed by the RBF network technique. Finally, based on the structure-loudness model, the robust optimum design for loudness was conducted.

\section{Design of structure samples and loudness calculation}

In order to elaborate the relation between the structure of exhaust system and the loudness of corresponding noise, several samples containing structure parameters of system and loudness of noise are necessary. The values of parameters of each sample are different from those of other samples. Considering that the engine speed is usually around $2000 \mathrm{r} / \mathrm{min}$, in the present study, the design of different samples was conducted in the condition that the engine speed is $2000 \mathrm{r} / \mathrm{min}$.

\subsection{Structure parameters of samples}

As previously mentioned, several parameters of a sample exhaust system which is shown in Fig. 1 should be selected. Considering the primary muffling element of exhaust noise is the silencer, the structure parameters of two silencers were chosen for samples. Owing to the pipe diameter and volume of chamber of silencer are the primary factors to influence the tail noise, eight parameters about them, described in Figs. 2 (a) and (b), were selected for the sake of giving a full description of the impact of structure parameters on the loudness of exhaust noise. These eight parameters are as follows: the diameter of inlet pipe of front silencer $\left(S_{1}\right)$, the diameter of outlet pipe 
of front silencer $\left(S_{2}\right)$, the diameter of inlet pipe of rear silencer $\left(S_{3}\right)$, the diameter of outlet pipe of rear silencer $\left(S_{4}\right)$, the diameter of perforation of silencer baffles $\left(S_{5}\right)$, the distance between baffle and outlet of front silencer $\left(S_{6}\right)$, the distance between first baffle and inlet of rear silencer $\left(S_{7}\right)$ and the distance between second baffle and inlet of rear silencer $\left(S_{8}\right)$.

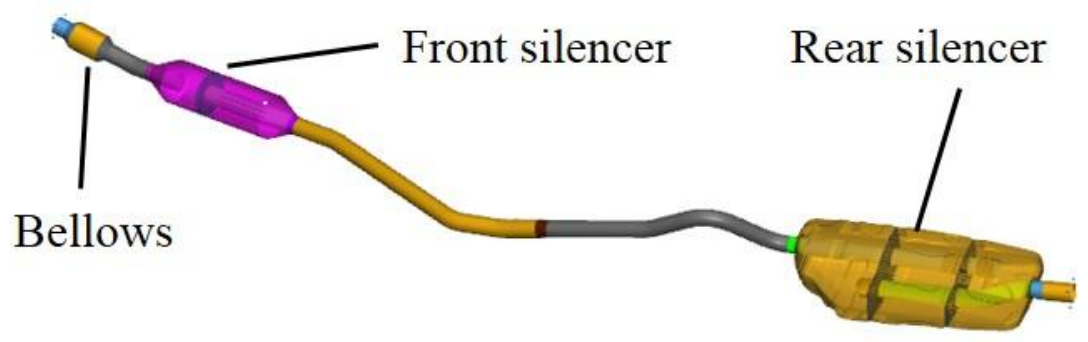

Fig. 1. Sample exhaust system

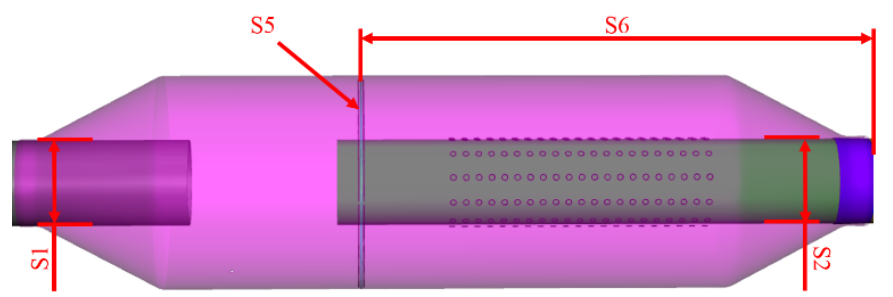

(a) Structure parameters of front silencer

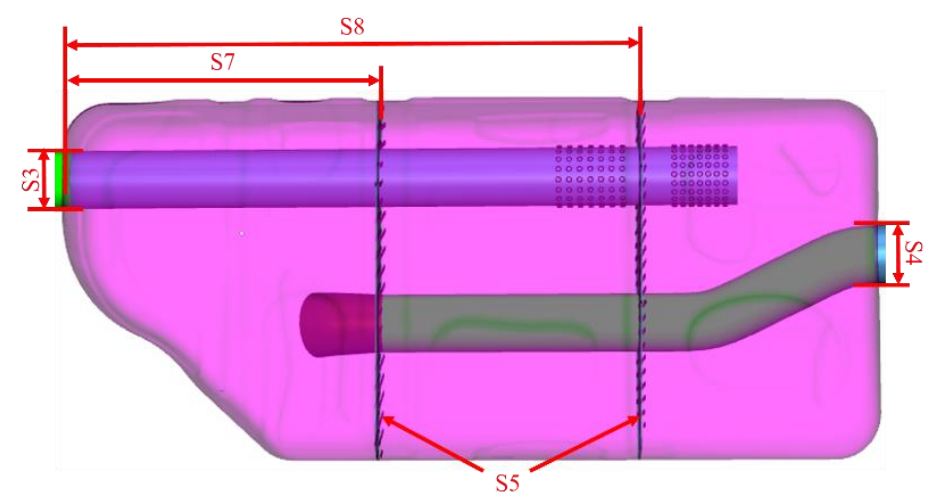

(b) Structure parameters of rear silencer

Fig. 2. Structure parameters of silencers

\subsection{Orthogonal design of samples}

After the structure parameters were determined, the orthogonal design was applied for the purpose of development of sample database. For the sake of better 
precision, more samples are required. However, more samples would increase the workload. Taking the both into consideration, a three-level orthogonal array, specifying 27 runs and having 8 columns, was employed in this work. The ranges and levels of eight selected parameters are shown in Table 1 and the detailed scheme of orthogonal design is listed in Table 2.

\section{Table 1}

Ranges and three levels of structure parameters

\begin{tabular}{ccccc}
\hline $\begin{array}{c}\text { Structure } \\
\text { parameters }(\mathrm{mm})\end{array}$ & Range & Level 1 & Level 2 & Level 3 \\
\hline$S_{1}$ & $42.3 \sim 51.7$ & 42.3 & 47 & 51.7 \\
$S_{2}$ & $43.2 \sim 52.8$ & 43.2 & 48 & 52.8 \\
$S_{3}$ & $38.34 \sim 46.86$ & 38.34 & 42.6 & 46.86 \\
$S_{4}$ & $38.34 \sim 46.86$ & 38.34 & 42.6 & 46.86 \\
$S_{5}$ & $3.15 \sim 3.85$ & 3.15 & 3.5 & 3.85 \\
$S_{6}$ & $237.2 \sim 296.5$ & 237.2 & 266.85 & 296.5 \\
$S_{7}$ & $245.4 \sim 294.48$ & 245.4 & 269.94 & 294.48 \\
$S_{8}$ & $400.86 \sim 489.94$ & 400.86 & 445.4 & 489.94 \\
\hline
\end{tabular}

Table 2

Orthogonal design of structure parameters

\begin{tabular}{ccccccccc}
\hline \multirow{2}{*}{ No } & \multicolumn{7}{c}{ Structure parameters(mm) } \\
\cline { 2 - 9 } & $S_{1}$ & $S_{2}$ & $S_{3}$ & $S_{4}$ & $S_{5}$ & $S_{6}$ & $S_{7}$ & $S_{8}$ \\
\hline 1 & 42.3 & 43.2 & 38.34 & 38.34 & 3.15 & 237.2 & 245.4 & 400.86 \\
2 & 42.3 & 43.2 & 42.6 & 42.6 & 3.5 & 266.85 & 269.94 & 445.4 \\
3 & 42.3 & 43.2 & 46.86 & 46.86 & 3.85 & 296.5 & 294.48 & 489.94 \\
4 & 42.3 & 48 & 38.34 & 42.6 & 3.5 & 296.5 & 294.48 & 445.4 \\
5 & 42.3 & 48 & 42.6 & 46.86 & 3.85 & 237.2 & 245.4 & 489.94 \\
6 & 42.3 & 48 & 46.86 & 38.34 & 3.15 & 266.85 & 269.94 & 400.86 \\
7 & 42.3 & 52.8 & 38.34 & 46.86 & 3.85 & 266.85 & 269.94 & 489.94 \\
8 & 42.3 & 52.8 & 42.6 & 38.34 & 3.15 & 296.5 & 294.48 & 400.86 \\
9 & 42.3 & 52.8 & 46.86 & 42.6 & 3.5 & 237.2 & 245.4 & 445.4 \\
10 & 47 & 43.2 & 38.34 & 42.6 & 3.85 & 266.85 & 294.48 & 400.86 \\
11 & 47 & 43.2 & 42.6 & 46.86 & 3.15 & 296.5 & 245.4 & 445.4 \\
12 & 47 & 43.2 & 46.86 & 38.34 & 3.5 & 237.2 & 269.94 & 489.94 \\
13 & 47 & 48 & 38.34 & 46.86 & 3.15 & 237.2 & 269.94 & 445.4 \\
14 & 47 & 48 & 42.6 & 38.34 & 3.5 & 266.85 & 294.48 & 489.94 \\
15 & 47 & 48 & 46.86 & 42.6 & 3.85 & 296.5 & 245.4 & 400.86 \\
16 & 47 & 52.8 & 38.34 & 38.34 & 3.5 & 296.5 & 245.4 & 489.94 \\
\hline
\end{tabular}




\begin{tabular}{ccccccccc}
\hline 17 & 47 & 52.8 & 42.6 & 42.6 & 3.85 & 237.2 & 269.94 & 400.86 \\
18 & 47 & 52.8 & 46.86 & 46.86 & 3.15 & 266.85 & 294.48 & 445.4 \\
19 & 51.7 & 43.2 & 38.34 & 46.86 & 3.5 & 296.5 & 269.94 & 400.86 \\
20 & 51.7 & 43.2 & 42.6 & 38.34 & 3.85 & 237.2 & 294.48 & 445.4 \\
21 & 51.7 & 43.2 & 46.86 & 42.6 & 3.15 & 266.85 & 245.4 & 489.94 \\
22 & 51.7 & 48 & 38.34 & 38.34 & 3.85 & 266.85 & 245.4 & 445.4 \\
23 & 51.7 & 48 & 42.6 & 42.6 & 3.15 & 296.5 & 269.94 & 489.94 \\
24 & 51.7 & 48 & 46.86 & 46.86 & 3.5 & 237.2 & 294.48 & 400.86 \\
25 & 51.7 & 52.8 & 38.34 & 42.6 & 3.15 & 237.2 & 294.48 & 489.94 \\
26 & 51.7 & 52.8 & 42.6 & 46.86 & 3.5 & 266.85 & 245.4 & 400.86 \\
27 & 51.7 & 52.8 & 46.86 & 38.34 & 3.85 & 296.5 & 269.94 & 445.4 \\
\hline
\end{tabular}

\subsection{Calculation of loudness}

After the acquirement of 27 groups which are shown in Table 2, numerical simulations were executed for the sound pressure levels in frequency domain. In the present work, the GT-POWER [21] was used to simulate the sound pressure level of each group. The sound pressure level of each group was used to calculate the loudness.

The loudness takes the filtering characteristic of human's auditory system into consideration, indicating the feeling of human's ear to the sound intensity of noise. In this work, the Zwicker's loudness method is used for computing the loudness of exhaust noise. The content of loudness calculation contains the specific loudness and total loudness. According to the standard ISO 532B [22], the calculation methods of specific loudness and total loudness are shown respectively in Eqs. (1) and (2):

$$
N^{\prime}=0.08\left(\frac{E_{\mathrm{TQ}}}{E_{0}}\right)^{0.23}\left[\left(0.5+0.5 \frac{E}{E_{\mathrm{TQ}}}\right)^{0.23}-1\right]
$$

where, $N^{\prime}$ is the specific loudness of the sound in sone/Bark; $E_{\mathrm{TQ}}$ is the excitation which is under the circumstance of the quiet ambient; $E_{0}$ is the excitation of a 
reference sound whose intensity is equal to $10^{-12} \mathrm{~W} / \mathrm{m}^{2} ; E$ is the excitation of the sound.

$$
N=\int_{0}^{24 \text { Bark }} N^{\prime} \mathrm{d} z
$$

where, $N$ is the total loudness of the sound and $z$ is the critical band rate in Bark.

The results of total loudness of 27 groups are shown in Table 3. Each group, containing the structure parameters and loudness, acts as a discrete sample of the whole sample database.

\section{Table 3}

Structure parameters and loudness

\begin{tabular}{|c|c|c|c|c|c|c|c|c|c|}
\hline \multirow{2}{*}{ No } & \multicolumn{8}{|c|}{ Structure parameters $(\mathrm{mm})$} & \multirow{2}{*}{ Loudness(sone) } \\
\hline & $S_{1}$ & $S_{2}$ & $S_{3}$ & $S_{4}$ & $S_{5}$ & $S_{6}$ & $S_{7}$ & $S_{8}$ & \\
\hline 1 & 42.3 & 43.2 & 38.34 & 38.34 & 3.15 & 237.2 & 245.4 & 400.86 & 20.63 \\
\hline 2 & 42.3 & 43.2 & 42.6 & 42.6 & 3.5 & 266.85 & 269.94 & 445.4 & 26.67 \\
\hline 3 & 42.3 & 43.2 & 46.86 & 46.86 & 3.85 & 296.5 & 294.48 & 489.94 & 32.1 \\
\hline 4 & 42.3 & 48 & 38.34 & 42.6 & 3.5 & 296.5 & 294.48 & 445.4 & 21.19 \\
\hline 5 & 42.3 & 48 & 42.6 & 46.86 & 3.85 & 237.2 & 245.4 & 489.94 & 31.39 \\
\hline 6 & 42.3 & 48 & 46.86 & 38.34 & 3.15 & 266.85 & 269.94 & 400.86 & 21.46 \\
\hline 7 & 42.3 & 52.8 & 38.34 & 46.86 & 3.85 & 266.85 & 269.94 & 489.94 & 24.29 \\
\hline 8 & 42.3 & 52.8 & 42.6 & 38.34 & 3.15 & 296.5 & 294.48 & 400.86 & 24.15 \\
\hline 9 & 42.3 & 52.8 & 46.86 & 42.6 & 3.5 & 237.2 & 245.4 & 445.4 & 28.03 \\
\hline 10 & 47 & 43.2 & 38.34 & 42.6 & 3.85 & 266.85 & 294.48 & 400.86 & 29.73 \\
\hline 11 & 47 & 43.2 & 42.6 & 46.86 & 3.15 & 296.5 & 245.4 & 445.4 & 27.54 \\
\hline 12 & 47 & 43.2 & 46.86 & 38.34 & 3.5 & 237.2 & 269.94 & 489.94 & 29.87 \\
\hline 13 & 47 & 48 & 38.34 & 46.86 & 3.15 & 237.2 & 269.94 & 445.4 & 26.17 \\
\hline 14 & 47 & 48 & 42.6 & 38.34 & 3.5 & 266.85 & 294.48 & 489.94 & 23.53 \\
\hline 15 & 47 & 48 & 46.86 & 42.6 & 3.85 & 296.5 & 245.4 & 400.86 & 27.35 \\
\hline 16 & 47 & 52.8 & 38.34 & 38.34 & 3.5 & 296.5 & 245.4 & 489.94 & 24.52 \\
\hline 17 & 47 & 52.8 & 42.6 & 42.6 & 3.85 & 237.2 & 269.94 & 400.86 & 32.04 \\
\hline 18 & 47 & 52.8 & 46.86 & 46.86 & 3.15 & 266.85 & 294.48 & 445.4 & 29.42 \\
\hline 19 & 51.7 & 43.2 & 38.34 & 46.86 & 3.5 & 296.5 & 269.94 & 400.86 & 28.07 \\
\hline 20 & 51.7 & 43.2 & 42.6 & 38.34 & 3.85 & 237.2 & 294.48 & 445.4 & 29.24 \\
\hline 21 & 51.7 & 43.2 & 46.86 & 42.6 & 3.15 & 266.85 & 245.4 & 489.94 & 31.97 \\
\hline 22 & 51.7 & 48 & 38.34 & 38.34 & 3.85 & 266.85 & 245.4 & 445.4 & 23.4 \\
\hline 23 & 51.7 & 48 & 42.6 & 42.6 & 3.15 & 296.5 & 269.94 & 489.94 & 28.26 \\
\hline 24 & 51.7 & 48 & 46.86 & 46.86 & 3.5 & 237.2 & 294.48 & 400.86 & 32.14 \\
\hline
\end{tabular}




\begin{tabular}{cccccccccc}
\hline 25 & 51.7 & 52.8 & 38.34 & 42.6 & 3.15 & 237.2 & 294.48 & 489.94 & 29.17 \\
26 & 51.7 & 52.8 & 42.6 & 46.86 & 3.5 & 266.85 & 245.4 & 400.86 & 29.81 \\
27 & 51.7 & 52.8 & 46.86 & 38.34 & 3.85 & 296.5 & 269.94 & 445.4 & 29.99 \\
\hline
\end{tabular}

\subsection{Experimental verification for simulation}

In order to guarantee the accuracy of subsequent investigation, an experiment was conducted to verify the accuracy of the loudness obtained by the simulation and calculation. The sample vehicle was equipped with testing exhaust system and worked on the revolving drum test table. The structure parameters of exhaust system in the experiment are shown in Table 4. The engine speed of vehicle is $2000 \mathrm{r} / \mathrm{min}$ which is the same as what we set in simulation. Regarding the measurement of exhaust noise, a high-fidelity microphone was prepared with the conditions specified in the standard ISO 5128 [23], as shown in Fig. 3.Because the loudness is computed by the sound pressure level of exhaust noise, we directly compare the sound pressure levels of the experiment and simulation. In this experiment, the frequency spectrum of exhaust noise was measured and compared with that of simulation. Considering that the low frequency noise plays a dominate role in exhaust noise, the low frequency spectrum of simulation and experiment was primarily compared, as expressed in Fig. 4. From Fig. 4 we can see that the result of simulation is in very good agreement with that of experiment. This has strongly validated the accuracy of the following analysis and proposed structure-loudness model which are very important to describe the relationship between the structure of exhaust system and loudness of noise.

\section{Table 4}

Structure parameters of exhaust system in experiment

\begin{tabular}{ccccccccc}
\hline $\begin{array}{c}\text { Structure } \\
\text { parameters }(\mathrm{mm})\end{array}$ & $S_{1}$ & $S_{2}$ & $S_{3}$ & $S_{4}$ & $S_{5}$ & $S_{6}$ & $S_{7}$ & $S_{8}$ \\
\hline Values & 47 & 48 & 42.6 & 42.6 & 3.5 & 296.5 & 245.4 & 445.4 \\
\hline
\end{tabular}




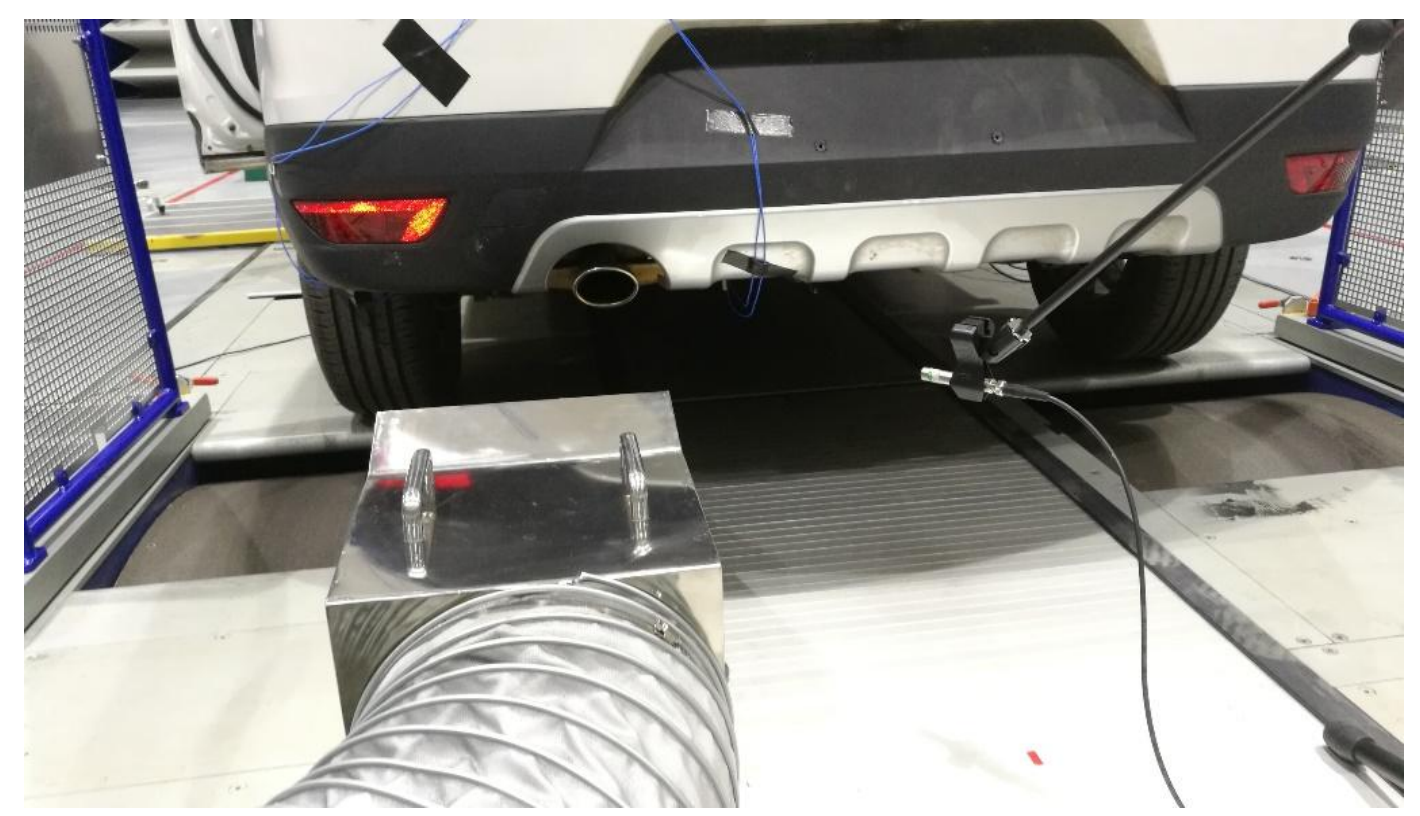

Fig. 3. The test table and microphone

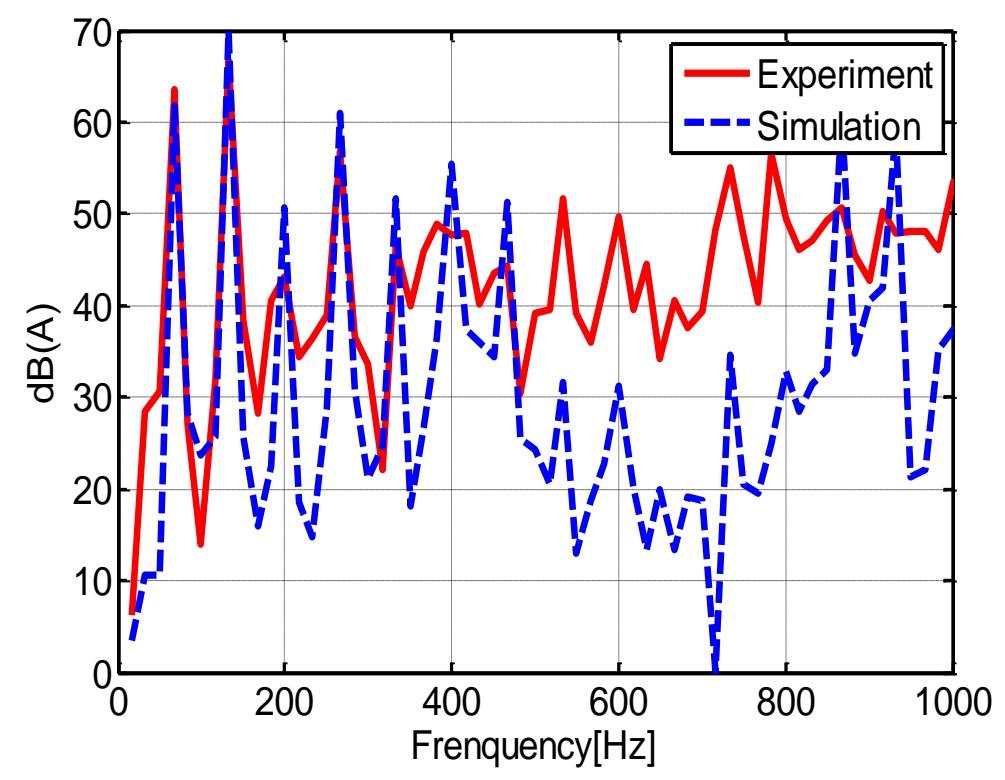

Fig. 4. Comparison result of simulation and experiment

\section{Analysis of impact of structure parameters on loudness}

\subsection{Pareto analysis}

The Pareto analysis is able to analyze the contribution of selected parameter and identify the dominant factor to the response, which can be very useful to the design of exhaust system. The Pareto analysis refers to the "80/20 rule" based on the observation about a pattern of "predictable imbalance" [24]. It asserts that the 80 
percent of resource is possessed by the 20 percent of the activity related to the resource, which means that the majority of the achievement is completed by the minority of the input. It was proved that the Pareto analysis, $80 / 20$ principle, can be applied in different disciplines [25]. Unfortunately, the previous researchers pay little attention to the application of the Pareto analysis to clarify the influence of structure to the sound quality evaluation. In this work, the contributions of structure parameters were conducted by the Pareto analysis to effectively reflect the impact on the loudness. The result of Pareto analysis is illustrated in Fig. 5. It can be found clearly that three significant factors which make primary contributions to the loudness are as follows: the diameter of inlet pipe of front silencer $\left(S_{1}\right)$, the diameter of inlet pipe of rear silencer $\left(S_{3}\right)$ and the diameter of outlet pipe of rear silencer $\left(S_{4}\right)$. Among these three primary factors, the maximal contribution is made by the diameter of inlet pipe of rear silencer $\left(S_{3}\right)$. The contributions of the diameter of inlet pipe of front silencer $\left(S_{1}\right)$ and the diameter of outlet pipe of rear silencer $\left(S_{4}\right)$ are both close to the consequence of the diameter of inlet pipe of rear silencer $\left(S_{3}\right)$. Besides, the diameter of perforation of silencer baffles $\left(S_{5}\right)$ and the distance between second baffle and inlet of rear silencer $\left(S_{8}\right)$ also make significant contributions. 


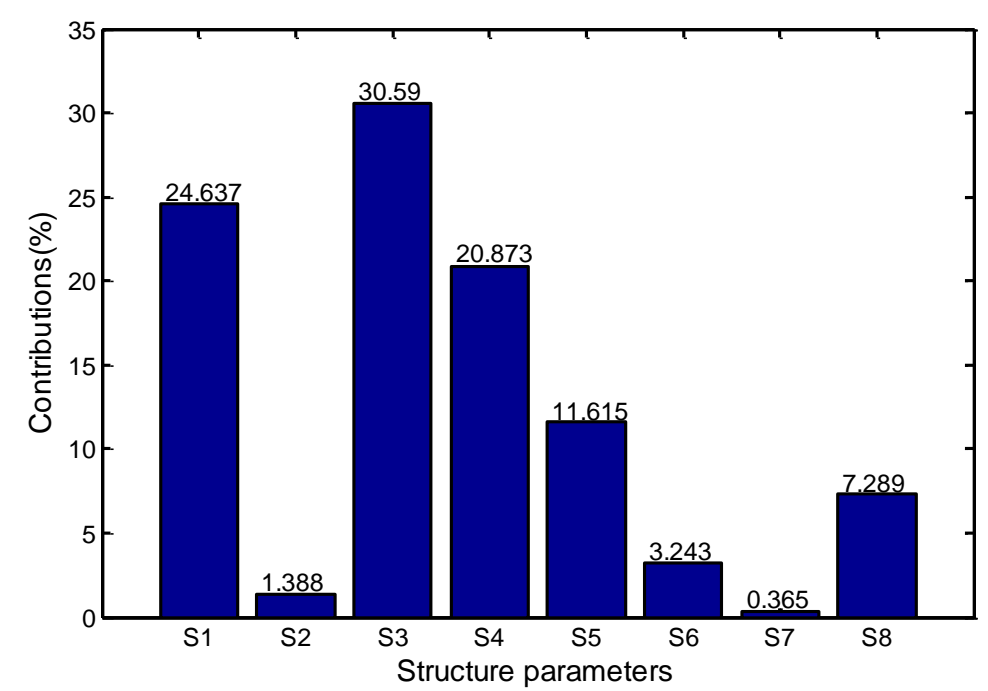

Fig. 5. Contributions of structure parameters on the loudness

\subsection{Main effects analysis}

The main effects analysis shows the variance of response when a factor or a group of factors changes among several levels, representing the sensitivities of factors to the response. For example, two factors, $M$ and $N$, which have two levels respectively with their responses, $R$, are exhibited as Table 5.

\section{Table 5}

Example factors and responses

\begin{tabular}{llll}
\hline$M$ & & $N$ & \\
& $N_{1}$ & $N_{2}$ \\
\hline $\mathrm{M}_{1}$ & $R_{11}$ & $R_{12}$ \\
$\mathrm{M}_{2}$ & $R_{21}$ & $R_{22}$ \\
\hline
\end{tabular}

The main effects of $M$ and $N, E_{M}$ and $E_{N}$, are computed as:

$$
\begin{aligned}
& E_{M}=\frac{\left(R_{21}-R_{11}\right)+\left(R_{22}-R_{12}\right)}{2} \\
& E_{N}=\frac{\left(R_{12}-R_{11}\right)+\left(R_{22}-R_{21}\right)}{2}
\end{aligned}
$$

In this work, the main effects of structure parameters on the loudness were calculated based on the variation of the single factor to reveal the separate effect of 
each structure parameter on loudness, as illustrated in Fig. 6. The slope of each line reveals the main effect of corresponding structure parameter. From Fig. 6 we can see that the diameter of inlet pipe of front silencer $\left(S_{1}\right)$, the diameter of inlet pipe of rear silencer $\left(S_{3}\right)$, the diameter of outlet pipe of rear silencer $\left(S_{4}\right)$, the diameter of perforation of silencer baffles $\left(S_{5}\right)$, the distance between first baffle and inlet of rear silencer $\left(S_{7}\right)$ and the distance between second baffle and inlet of rear silencer $\left(S_{8}\right)$ show positive effects. But the diameter of outlet pipe of front silencer $\left(S_{2}\right)$ and the distance between baffle and outlet of front silencer $\left(S_{6}\right)$ show negative effects. The loudness increases when the structure parameters with positive effects increase while it decreases when the negative parameters increase. Among all of them, the diameter of outlet pipe of rear silencer $\left(S_{4}\right)$ has the biggest effect on the loudness, indicating that the loudness is the most sensitive to it. Besides, the sensitivity of the distance between first baffle and inlet of rear silencer $\left(S_{7}\right)$ is the same as that of the distance between second baffle and inlet of rear silencer $\left(S_{8}\right)$.

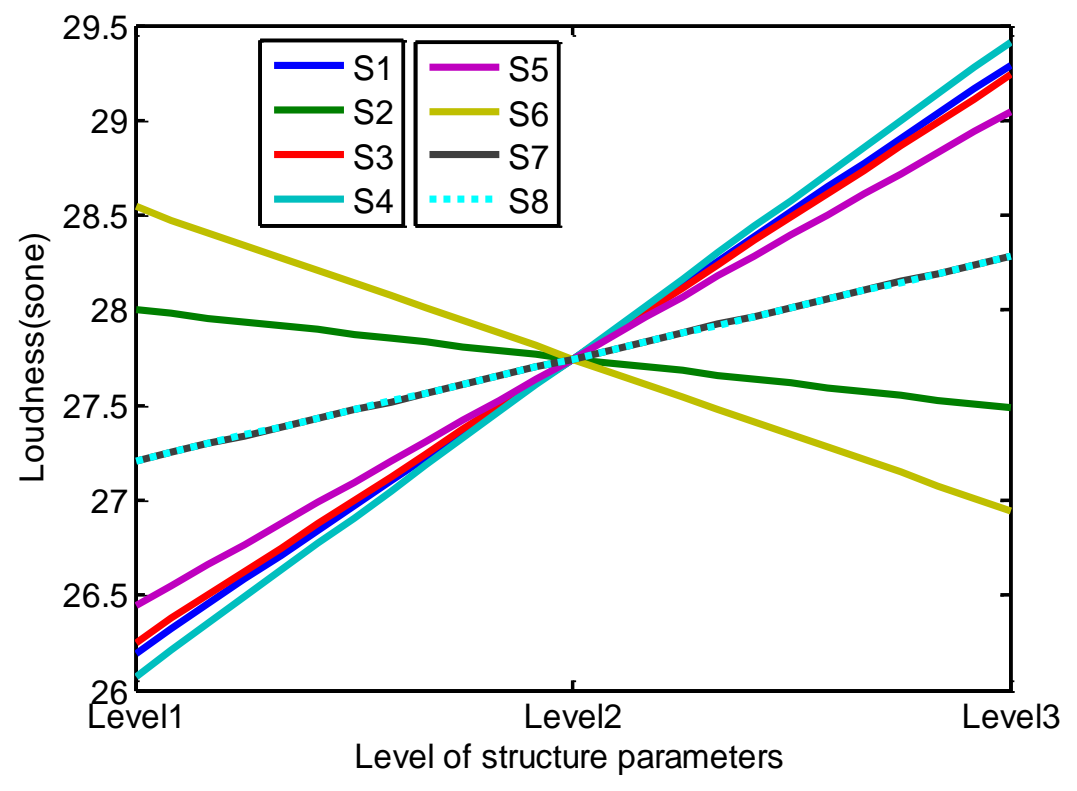

Fig. 6. Main effects of structure parameters to loudness 


\section{Development of structure-loudness model}

\subsection{Theory of RBF network}

Because of the nonlinear relation between the structure of exhaust system and the sound quality of noise, a nonlinear sound quality model need be developed for better perception of the sound. The RBF network is characterized by reasonably fast training and compact network. It does a good job to approximate the wide range of nonlinear space, very suitable for the development of this model. The RBF network is a type of neural network which contains an input layer, a hidden layer for radial units and an output layer [26].

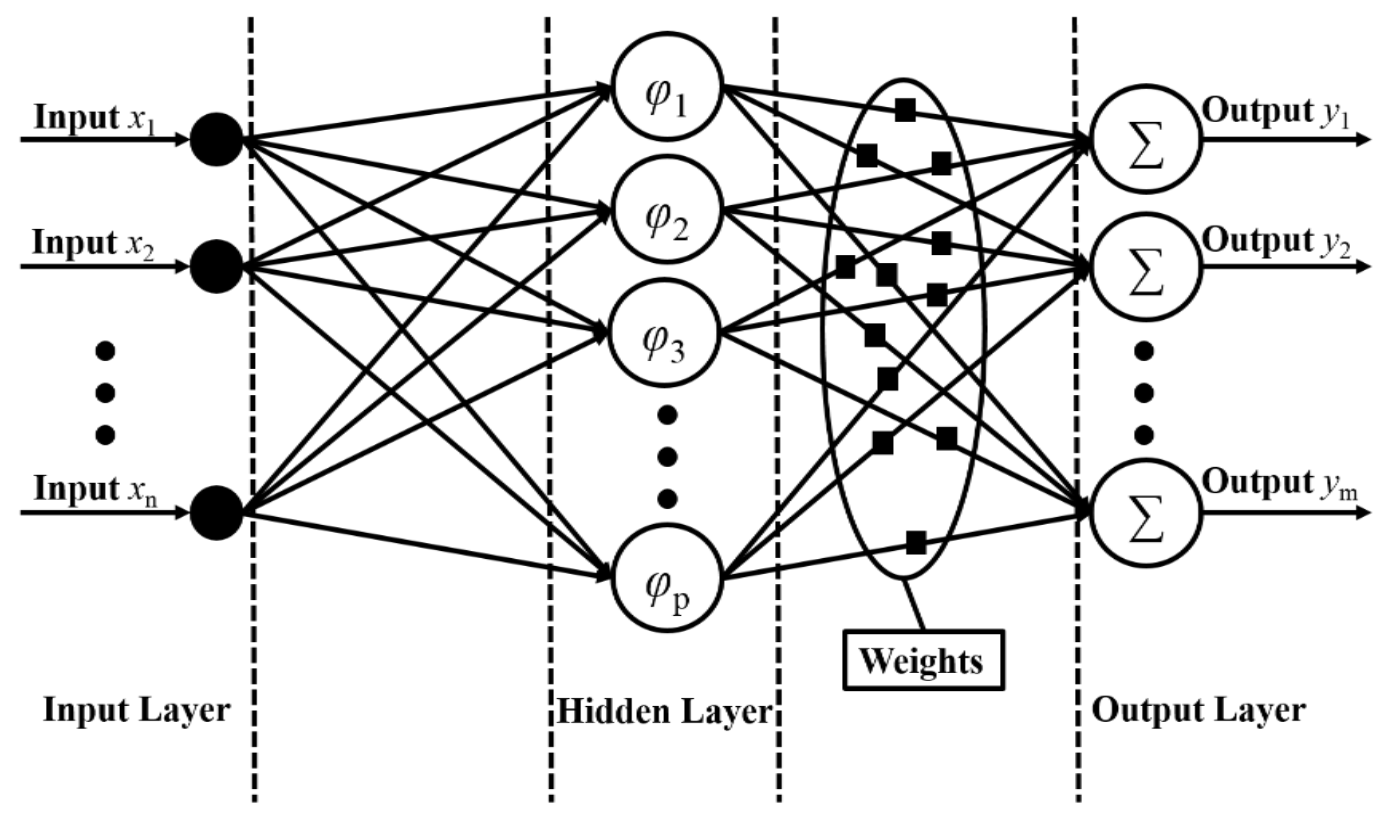

Fig. 7. Radial basis function network

The construction of RBF network is shown in Fig. 7. The output of RBF network is equal to the sum of weighted radial functions as expressed in Eq. (5):

$$
\mathbf{F}(\mathbf{X})=\sum_{i=1}^{p} w_{i} \varphi_{i}\left(\left\|\mathbf{X}-\mathbf{X}_{i}\right\|\right)
$$

where $\mathbf{F}(\mathbf{X})$ is the output vector of RBF network; $p$ is the number of samples; $w_{i}$ is the 
weighted coefficient of the radial basis function of the $i$ th sample; $\varphi_{i}$ is the radial basis function of the $i$ th sample; $\mathbf{X}$ is the $n$-dimensional input vector, shown as Eq. (6); $\mathbf{X}_{i}$ is the n-dimensional input vector of the $i$ th sample; $\left\|\mathbf{X}-\mathbf{X}_{i}\right\|$ is the Euclidean distance between $\mathbf{X}$ and $\mathbf{X}_{i}$.

$$
\mathbf{X}=\left\{x_{1}, x_{2}, \cdots, x_{\mathrm{n}}\right\}
$$

where $x_{1}, x_{2}, \cdots, x_{\mathrm{n}}$ are the input factors shown in Fig. 7.

$$
\mathbf{Y}=\left\{y_{1}, y_{2}, \cdots, y_{\mathrm{m}}\right\}
$$

where $\mathbf{Y}$ is the m-dimensional vector; $y_{1}, y_{2}, \cdots, y_{\mathrm{m}}$ are the output factors shown in Fig. 7.

The output factors of samples can be integrated as an $m$-dimensional vector as Eq. (7). In Eq. (5), the output vectors of RBF network should be equal to the counterparts of samples, as expressed in Eq. (8).

$$
\mathbf{F}\left(\mathbf{X}_{i}\right)=\mathbf{Y}_{i}, \quad i=1,2, \cdots, \mathrm{p}
$$

where $\mathbf{F}\left(\mathbf{X}_{i}\right)$ is the output vector of RBF network whose the input vector is $\mathbf{X}_{i} ; \mathbf{Y}_{i}$ is the output vector of the $i$ th sample.

In this work, the Gaussian function, expressed in Eq. (9), was set as the radial basis function.

$$
\varphi_{i}(r)=e^{-\frac{r^{2}}{2 c^{2}}}
$$

where $r$ is the input of the Gaussian function; $c$ is the constant value of this function 
which is predefined.

Eqs. (5) and (9) show the formulation of RBF network. Eq. (8) shows the constraints of RBF network. In this work, the RBF network was trained and implemented by the software of iSIGHT [27].

\subsection{Development of structure-loudness model}

In this work, sample database was utilized to approximate the relationship between structure parameters and loudness by the RBF network to develop the structure-loudness model. The RBF network training procedure was performed in iSIGHT. After a great number of training tests, the constitution of the structure-loudness model was determined and shown in Figs. 8(a)-(d). This structure-loudness model can be directly used to predict the loudness of exhaust noise when the values of structure parameters are determinate and conduct an optimization.

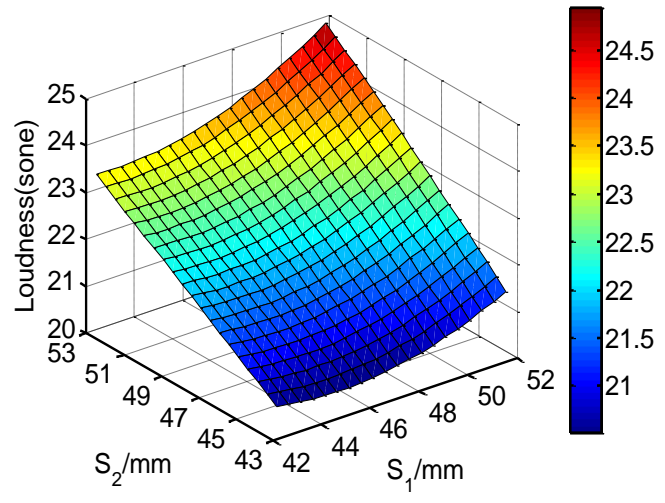

(a) Loudness vs. $S_{1}$ and $S_{2}$

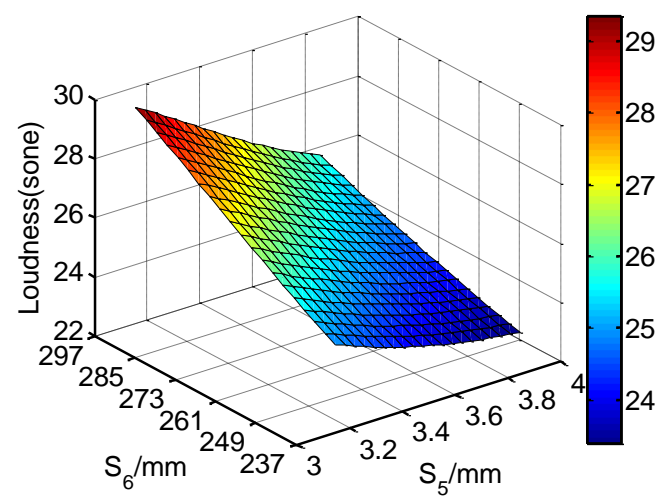

(c) Loudness vs. $S_{5}$ and $S_{6}$

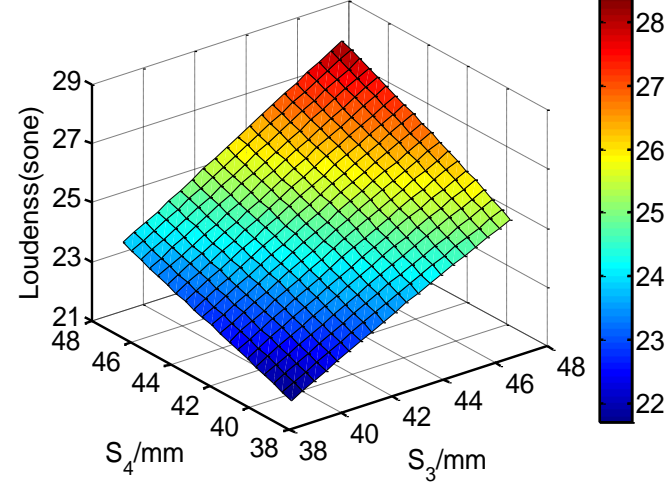

(b) Loudness vs. $S_{3}$ and $S_{4}$

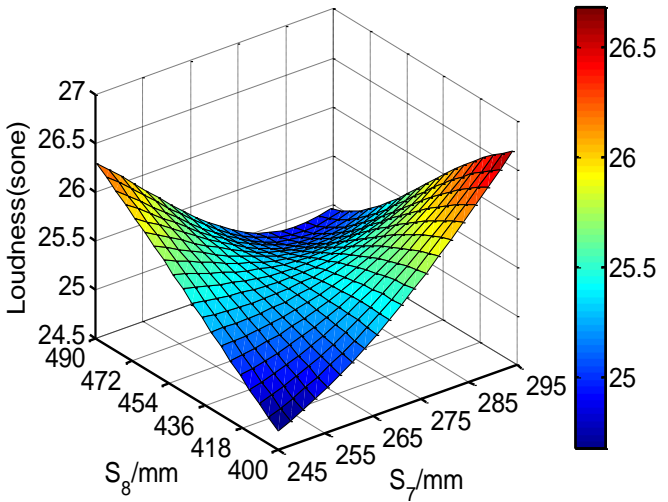

(d) Loudness vs. $S_{7}$ and $S_{8}$ 
Fig. 8. Constitution of structure-loudness model

\section{Uncertainty-based optimum design for loudness}

\subsection{Stochastic model of structure parameters}

The stochastic model is an important method to deal with the uncertainty problem. The probability density function is used to reflect the output of stochastic model on the basis of enough samples. All samples are gathered as an aggregate, as shown in Eq (10).

$$
\mathbf{D}=\left\{\mathbf{D}_{1}, \mathbf{D}_{2}, \cdots, \mathbf{D}_{u}\right\}
$$

where $\mathbf{D}$ is the aggregate of samples; $\mathbf{D}_{1}, \mathbf{D}_{2}, \cdots, \mathbf{D}_{u}$ are the samples; $u$ is the number of samples.

Each sample containing several variables can be defined as the $m$-dimensional vector as $\mathrm{Eq}(11)$.

$$
\mathbf{D}_{i}=\left\{d_{i 1}, d_{i 2}, \cdots, d_{i v}\right\}, \quad i=1,2, \cdots, u
$$

where $\mathbf{D}_{i}$ is the $i$ th sample; $d_{i 1}, d_{i 2}, \cdots, d_{i v}$ are the variables of the $i$ th sample; $v$ is the number of the variables of the $i$ th sample.

The probability density function is described by the mean value, standard deviation and coefficient of variation, as illustrated in Eqs. (12) - (14) respectively.

$$
\mu_{j}=\frac{\sum_{i=1}^{u} d_{i j}}{u}, \quad j=1,2, \cdots, v
$$

where $\mu_{j}$ is the mean value of the $j$ th variable; $d_{i j}$ is the $j$ th variable of the $i$ th sample. 


$$
\sigma_{j}=\sqrt{\frac{\sum_{i=1}^{u}\left(d_{i j}-\mu_{j}\right)^{2}}{u}}
$$

where $\sigma_{j}$ is the standard deviation of the $j$ th variable.

$$
C V_{j}=\frac{\sigma_{j}}{\mu_{j}}
$$

where $C V_{j}$ is the coefficient of variation of the $j$ th variable.

Considering that the error of structure happens normally in the manufacture, we assumed that the distribution of each structure parameter is the normal distribution and the probability density function of normal distribution is showed in Eq. (15).

$$
f\left(d_{j}\right)=\frac{1}{\sqrt{2 \pi} \sigma_{j}} e^{-\frac{\left(d_{j}-\mu_{j}\right)^{2}}{2 \sigma_{j}^{2}}}, \quad-\infty<d_{j}<\infty
$$

Where $f\left(d_{j}\right)$ is the probability density function of normal distribution; $d_{j}$ is the $j$ th variable of the samples.

In the robust optimum design, the stochastic models of structure parameters were used to represent the uncertainty of system. In the present work, both the optimum mean value and standard deviation should be paid attention. The mean values and standard deviations of different parameters were maintained within normal distribution, as shown in Table 6. In order to obtain the optimum represent and robustness of loudness, the minimums of mean value and standard deviation were set as the objectives of the optimization. With a view to the possibility that the optimal values of mean and standard deviation cannot be obtained at the same time, we give 
different weighting coefficients for them. The optimization objective is expressed as:

$$
\min F=\omega_{1} \mu_{\mathrm{SQM}}+\omega_{2} \sigma_{\mathrm{SQM}}
$$

Here, $F$ is the optimal objective function; $\omega_{1}$ and $\omega_{2}$ are respectively the weighting coefficients of the mean value and standard deviation of the sound quality evaluation; $\mu_{\mathrm{SQM}}$ is the mean value of the sound quality evaluation; $\sigma_{\mathrm{SQM}}$ is the standard deviation of the sound quality metric.

\section{Table 6}

Uncertainty distribution information

\begin{tabular}{ccccccccc}
\hline \multirow{2}{*}{$\begin{array}{c}\text { Uncertainty } \\
\text { distribution }\end{array}$} & \multicolumn{8}{c}{ Structure parameters } \\
\cline { 2 - 9 } & $S_{1}$ & $S_{2}$ & $S_{3}$ & $S_{4}$ & $S_{5}$ & $S_{6}$ & $S_{7}$ & $S_{8}$ \\
\hline Mean & 47 & 48 & 42.6 & 42.6 & 3.5 & 266.85 & 269.94 & 445.4 \\
$\begin{array}{c}\text { Standard } \\
\text { deviation }\end{array}$ & 2.115 & 2.16 & 1.917 & 1.917 & 0.1575 & 11.86 & 12.27 & 20.043 \\
\hline
\end{tabular}

\subsection{Review of robust optimum design method}

\subsubsection{Six sigma design}

The consistent performance of product is always desired in industry. To ensure this consistency, the quality of product is measured and improved [28]. In the manufacture, the quality of production is affected by the uncertainty of structure. In the research of quality control engineering, the six sigma, proposed by Bill Smith in 1986, is often used to control and improve the robustness. In the six sigma design, the standard deviation, $\sigma$, is used to perform the variability of factors with normal distribution and the performance variation is characterized as several standard deviations from the mean value, $\mu$, as shown in Fig. 9. The area under the normal distribution is associated with the whole probability of performance. The lower and 
upper limits define the desirable range of the whole probability in accordance with $\pm 6 \sigma$ from the $\mu$. The probabilities of each $\sigma$-level fell in particular range and are expressed as percent variation in Table 7.

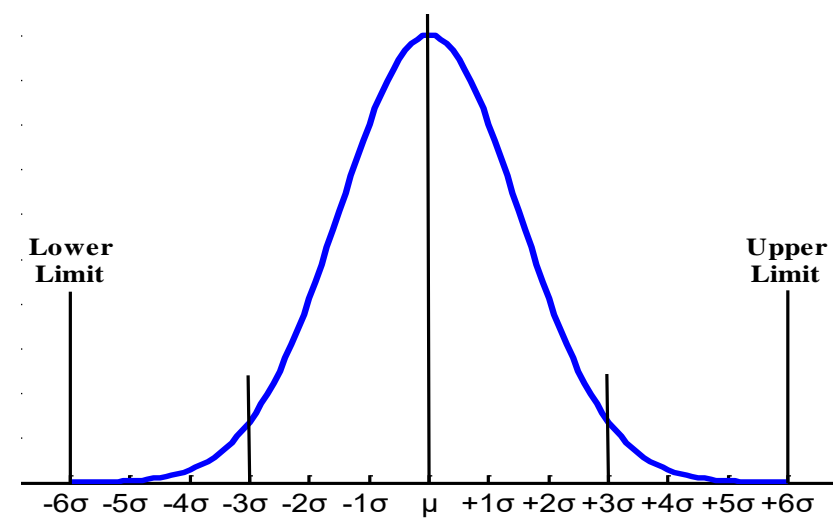

Fig. 9. Performance variation of six sigma design

Table 7

Percent variation of sigma level

\begin{tabular}{ccccccc}
\hline $\begin{array}{c}\text { Sigma } \\
\text { level }\end{array}$ & $\pm 1 \sigma$ & $\pm 2 \sigma$ & $\pm 3 \sigma$ & $\pm 4 \sigma$ & $\pm 5 \sigma$ & $\pm 6 \sigma$ \\
\hline $\begin{array}{c}\text { Percent } \\
\text { variation }\end{array}$ & 68.26 & 95.46 & 99.73 & 99.9937 & 99.999943 & 99.999998 \\
\hline
\end{tabular}

In order to obtain products with high-quality, the six sigma design strives to maintain the percent variation of $\pm 6 \sigma$ level within the limitation can be accepted, i.e., the probability of performance remaining within the set limits should be essentially $100 \%$.

In this work, the Six Sigma quality control mode was used to limit the deviation of the loudness for the best robustness, which was executed in iSIGHT. As an important part of Six Sigma control, the Monte Carlo sampling is a very efficient technique for reduction of variance. In this work, the Monte Carlo sampling was utilized to choose a set of sample points from the structure-loudness model and its 
sampling technique was simple random sampling. Because the calculated workload would increase with the increasing of the amount of sample points, the number of sample points was limited and set as 25 .

\subsubsection{Adaptive simulated annealing algorithm}

Regarding to the optimum design of exhaust system, the choice of appropriate algorithm is crucial for the successful optimization. The adaptive simulated annealing (ASA) algorithm has the advantage that it is well suitable for non-linear system solution with short running analysis codes which leads to a quick improvement for the design. In this study, the ASA algorithm was used to the robust optimization of exhaust system for improved loudness. The ASA algorithm is a stochastic search method that its iteration is similar to the annealing process from statistical mechanics and metallurgy. This algorithm follows a "cooling schedule" until steady state or the optimal solution is obtained. The structure of ASA algorithm is mainly comprised of three parts: the generation of probability density function, the acceptance of probability density function and annealing temperature schedule.

ASA considers the optimal parameter $\alpha_{k}$ with a constraint range at the annealing-time $k$ as:

$$
\alpha_{k} \in[A, B], \quad k=1,2, \cdots
$$

where $A$ and $B$ are the lower and upper bounds respectively.

In each iteration, two main steps, generating a candidate solution and determining if the solution is accepted, are conducted. A candidate solution $\alpha_{k+1}$ is calculated from the old parameter $\alpha_{k}$ with a random variable $y$, as shown in Eq. (18). 


$$
\alpha_{k+1}=\alpha_{k}+y(B-A), \quad y \in[-1,1]
$$

The new solution is generated within a distribution defined by the probability density function, as illustrated in Eq. (19).

$$
g\left(y, T_{k}\right)=\frac{1}{2\left(|y|+T_{k}\right) \ln \left(1+\frac{1}{T_{k}}\right)}
$$

where $T_{k}$ is the temperature of $k$ th iteration and the annealing temperature schedule specifies that it is related to the initial temperature $T_{0}$ as Eq. (20).

$$
T_{k}=T_{0} \exp (-c k)
$$

where $\mathrm{c}$ is an exponent of annealing temperature schedule.

The acceptance of new solution is determined by the cost function, the cost temperature and a uniform random generator. New solution is accepted for next iteration if

$$
\exp \left[-\frac{C\left(\alpha_{k+1}\right)-C\left(\alpha_{k}\right)}{T_{\text {cost }}}\right]>U, \quad U \in[0,1)
$$

where $C\left(\alpha_{k+1}\right)-C\left(\alpha_{k}\right)$ is the cost function; $T_{\text {cost }}$ is the cost temperature; $U$ is the uniform random generator.

\subsection{Uncertainty-based optimization for loudness}

As inferred above, $\mu$ is the mean value of the objective and $6 \sigma$ is the quality measurement of the uncertainty in six sigma quality design. In this work, $\mu$ and $6 \sigma$ represent the magnitude and deviation of the obtained result of loudness respectively. For the purpose of lower loudness and higher accuracy of consequence, both the $\mu$ and $6 \sigma$ should be decreased. Therefore, the $\omega_{1}$ and $\omega_{2}$ were set to 1 and 6 respectively 
and the objective function of this optimization is described as:

$$
\min F=\mu_{\mathrm{SQM}}+6 \sigma_{\mathrm{SQM}}
$$

Based on the optimization method above, the trace of best solution of objective minimization is obtained, as shown in Fig. 10. From this figure we can see that the iteration of optimal value exhibits fast convergence as a result of the usage of adaptive simulated annealing algorithm. The value of objective function changes rapidly in the first 20 iterations and is minimized in the $100^{\text {th }}$ iteration. The optimum results of the mean value and standard deviation of the loudness and the structure parameters corresponding to the optimum response are respectively illustrated in Table 8. As can be seen that after the optimization, the mean of loudness is 21.83 and the standard deviation is 1.06. These optimization results are so obvious that the robust optimum design method can be put into the practical use.

\section{Table 8}

Optimization result of loudness

\begin{tabular}{cccccccccc}
\hline \multicolumn{1}{c}{ Inputs } & & \multicolumn{2}{c}{ Outputs } \\
\hline \multicolumn{1}{c}{ Structure parameter } & & & \multicolumn{2}{c}{ Loudness } \\
$S_{1}$ & $S_{2}$ & $S_{3}$ & $S_{4}$ & $S_{5}$ & $S_{6}$ & $S_{7}$ & $S_{8}$ & Mean & $\begin{array}{c}\text { Standard } \\
\text { Deviation }\end{array}$ \\
\hline 43.27 & 52.06 & 38.46 & 39.24 & 3.24 & 267.78 & 267.49 & 452.71 & 21.83 & 1.06 \\
\hline
\end{tabular}




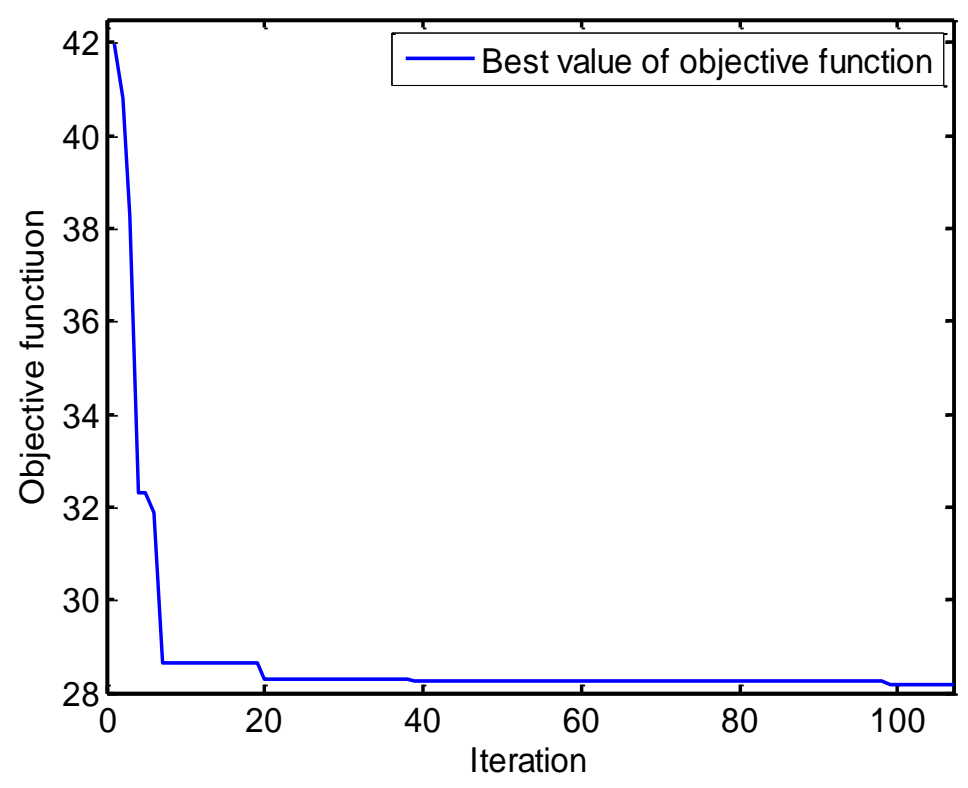

Fig. 10. Trace of objective minimization over iteration

\section{Conclusions}

In this paper, the influence of structure parameters of the exhaust system on the loudness of exhaust tail noise, and the relationship between the parameters and loudness were analyzed. Based on the structure-loudness model developed in this work, a robust optimum design of exhaust system was conducted. Then, a three-level orthogonal array for all parameters was designed and the orthogonal design method was used to formulate twenty-seven discrete sample data groups. The sound pressure level of exhaust tail noise of each group was simulated and the corresponding loudness was calculated. The verification experiment was conducted to demonstrate the feasibility of the simulation. Based on the sample data above, the contributions and the main effects of structure parameters on the loudness of noise were analyzed, indicating that the greatest contribution of loudness is made by the diameter of inlet pipe of rear silencer. Meanwhile, the main effects analysis has shown that the diameter of outlet pipe of rear silencer has a primary effect on the loudness. Moreover, 
the RBF network was used to develop the structure-loudness model. For the application of this model, the robust optimization of the loudness with the consideration of uncertainty of structure was completed by the adaptive simulated annealing (ASA) algorithm. In view of the optimization results, both the mean value and the standard deviation of the loudness were optimized. Thus, the structure-loudness model is able to offer an efficient tool to optimize the loudness of the exhaust system.

\section{Acknowledgements}

This work was supported by SAIC-GM-Wuling Automobile cooperation and Science Fund of State Key Laboratory of Advanced Design and Manufacturing for Vehicle Body Nos. 51375001.

\section{Reference}

[1] Brandl, F. K., and Biermayer, W., 1999, "A New Tool for the Onboard Objective Assessment of Vehicle Interior Noise Quality," Papers;Automotive_Sector.

[2] Trapenskas, D., 2016, "Sound quality assessment using binaural technology," Luleå Tekniska Universitet.

[3] Murata, H., Tanaka, H., Takada, H., and Ohsasa, Y., "Sound Quality Evaluation of Passenger Vehicle Interior Noise," Proc. Noise \& Vibration Conference \& Exposition.

[4] Volandri, G., Puccio, F. D., Forte, P., and Mattei, L., 2018, "Psychoacoustic analysis of power windows sounds: Correlation between subjective and objective evaluations," Applied Acoustics, 134, pp. 160-170.

[5] Kwon, G., Jo, H., and Kang, Y. J., 2018, "Model of psychoacoustic sportiness for vehicle interior sound: Excluding loudness," Applied Acoustics, 136, pp. 16-25.

[6] Singh, S., and Mohanty, A. R., 2018, "HVAC noise control using natural materials to improve vehicle interior sound quality," Applied Acoustics, 140, pp. 100-109.

[7] Jekosch, U., 1997, "Meaning in the Context of Sound Quality Assessment," Acta Acustica United with Acustica, 85(5), pp. 681-684.

[8] Lighthill, M. J., 1952, "On Sound Generated Aerodynamically. I. General Theory," Proceedings of the Royal Society of London, 211(1107), pp. 564-587.

[9] Zhao, S., Shang, C., Zhao, Z., and Shi, W., 2000, "Radiation characteristics of intermittence exhaust noise," Chinese Journal of Acoustics(4), pp. 309-316.

[10] Zhao, S., Wang, J., Wang, J., and He, Y., 2006, "Expansion-chamber muffler for impulse noise of 
pneumatic frictional clutch and brake in mechanical presses," Applied Acoustics, 67(6), pp. 580-594.

[11] Shi, H., and Zhao, S., 2009, "Prediction of radiation characteristic of intermittent exhaust noise generated via pneumatic value," Noise Control Engineering Journal, 57(3), pp. 157-168(112).

[12] Li, J., Ishihara, K., and Zhao, S., "Experimental study on performance of various mufflers for intermittent exhaust noise reduction," pp. 1706-1711(1706).

[13] Villot, M., Guigou, C., and Gagliardini, L., 2001, "PREDICTING THE ACOUSTICAL RADIATION OF FINITE SIZE MULTI-LAYERED STRUCTURES BY APPLYING SPATIAL WINDOWING ON INFINITE STRUCTURES," Journal of Sound \& Vibration, 245(3), pp. 433-455.

[14] Li, J., Zhao, S., and Ishihara, K., 2013, "Study on acoustical properties of sintered bronze porous material for transient exhaust noise of pneumatic system," Journal of Sound \& Vibration, 332(11), pp. 2721-2734.

[15] Ranjbar, M., "On Muffler Design for Transmitted Noise Reduction," Proc. International Symposium on Multidisciplinary Studies and Innovative Technologies.

[16] Zhang, Y. Y., Li, S. M., Hu, Y. X., Hu, G. Y., Lu, W. F., and Wang, X. X., 2011, "Experimental Analysis and CAE Optimization for Exhaust Noise of the Diesel," Applied Mechanics \& Materials, 105-107, pp. 366-369.

[17] Šteblaj, P., Čudina, M., Lipar, P., and Prezelj, J., 2015, "Adaptive muffler based on controlled flow valves," Journal of the Acoustical Society of America, 137(6), pp. 503-509.

[18] Apley, D. W., Liu, J., and Chen, W., 2016, "Understanding the Effects of Model Uncertainty in Robust Design With Computer Experiments," Journal of Mechanical Design, 128(4), pp. 1183-1192.

[19] Zhang, Y., Li, M., Zhang, J., and Li, G., 2016, "Robust optimization with parameter and model uncertainties using Gaussian processes," Journal of Mechanical Design, 138(11), p. V02BT03A049.

[20] Son, Y. K., and Savage, G. J., 2016, "Stability and robustness analysis of uncertain nonlinear systems using entropy properties of left and right singular vectors," Journal of Mechanical Design, 139(3).

[21] Zehni, A., and Hossainpur, S., 2014, "Optimizing exhaust noise and back pressure of exhaust system in turbo-charged three-liter diesel engine," International Journal of Engine Research.

[22] Isotc, A., 1975, "ACOUSTICS - METHOD FOR CALCULATING LOUDNESS LEVEL. FIRST EDITION."

[23] 1980, "ISO 5128:1980-08 Acoustic-Measurement of noise inside motor vehicles."

[24] Taman, P., and Tanya, S. B., 2015, Pareto analysis, John Wiley \& Sons, Ltd.

[25] 1997, "The 80/20 Principle: The Secret of Achieving More With Less."

[26] Hossain, M. S., Chao, O. Z., Ismail, Z., and Yee, K. S., 2017, "A Comparative Study of Vibrational Response Based Impact Force Localization and Quantification Using Radial Basis Function Network and Multilayer Perceptron," Expert Systems with Applications.

[27] Park, K. B., Chung, W. J., and Lee, C. M., 2009, "A Study on Roughness Characteristic about Rotational Accuracy Variation," Journal of the Korean Society of Manufacturing Technology Engineers, 18(5), pp. 110-115.

[28] Koch, P. N., Yang, R. J., and Gu, L., 2004, "Design for six sigma through robust optimization," Structural \& Multidisciplinary Optimization, 26(3-4), pp. 235-248. 